\title{
EL IMPERIO DE LA LEY Y LA INTERPRETACIÓN Y APLICACIÓN DEL DERECHO: DOS VISIONES
}

\author{
Juan Carlos Bayón \\ Universidad Autónoma de Madrid
}

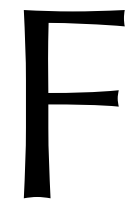

rancisco LAPORTA ha sostenido que «la mejor visión» de los procesos de interpretación y aplicación del derecho, dadas las «exigencias implícitas en el ideal regulativo del imperio de la ley», es la que reivindica «el textualismo y el literalismo formalista» (LAPORTA, 2007: 192). En estas páginas me propongo sugerir dos cosas. En primer lugar, que a esa visión cabe contraponerle otra que en mi opinión resulta preferible. $Y$, en segundo lugar, que en realidad esa visión alternativa no debería considerarse completamente ajena al pensamiento de Francisco LAPORTA ni al de Liborio HIERRO. Avanzando por el camino que nos abrió Elías DíAz, HIERRO y LAPORTA han ido elaborando en las últimas décadas las mejores reflexiones producidas entre nosotros acerca del ideal del imperio de la ley y su trascendencia para nuestra concepción del derecho y su aplicación, con un notable grado de coincidencia y también, cuando esa coincidencia no es plena, de provechosa complementariedad. Y como digo, creo que en sus escritos cabe rastrear las piezas que permitirían articular esa visión alternativa, que, no obstante, queda en ellos ensombrecida por aquella otra que se defiende de manera expresa.

1. El itinerario argumental que sigue LAPORTA en su reivindicación del literalismo formalista es bien conocido. El imperio de la ley, como ideal, tendría su fundamento en el valor de la autonomía. Pero la autonomía personal, la capacidad de trazar y seguir planes de vida, no es posible sin expectativas sólidas acerca de la conducta de los demás (incluidas sus reacciones a nuestras conductas), así que la autonomía requiere predecibilidad. Y lo que hace posible la predecibilidad es la existencia de normas y singularmente de normas jurídicas, pero no de cualquier tipo, ni interpretadas y aplicadas de cualquier modo. La predecibilidad requeriría normas formuladas como reglas (que correlacionen casos genéricos — configurados mediante una combinación de propiedades - con soluciones igualmente genéricas), dotadas de autonomía semántica (que no configuren el caso genérico ni su solución normativa - la acción modalizada deónticamente - mediante propiedades valorativas), interpretadas literalmente (de acuerdo con el significado ordinario de las formulaciones normativas, que es determinado por convenciones lingüísticas y no atribuido por el intérprete ni dependiente de la intención del emisor) y aplicadas de manera formalista (subsumiendo sin más los casos individuales en el caso genérico regulado, sin entrar a considerar las razones subyacentes a las reglas para corregir posibles casos de supra-inclusión o infra-inclusión). Porque, de lo contrario, entre las normas y 
las decisiones de los aplicadores podría haber tantas interferencias que difícilmente podría decirse que las segundas están gobernadas realmente por las primeras. Y con ello quedaría comprometida la predecibilidad, requerida por el valor fundamental de la autonomía ${ }^{1}$.

Lo que ocurre - como ya han señalado distintas voces críticas- es que ese razonamiento encierra dos pasos problemáticos. Simplificado al extremo, el argumento sostiene que la autonomía requiere predecibilidad y que la predecibilidad requiere un derecho de reglas aplicadas de manera formalista. Para empezar, puede que el segundo de estos pasos no sea tan seguro, si se acepta que un derecho de reglas aplicadas de manera formalista no es realmente condición necesaria ni suficiente de la predecibilidad ${ }^{2}$. Creo que en rigor efectivamente es así, aunque también me parece difícil negar que, si todo lo que importara fuese la predecibilidad, entre las distintas opciones imaginables la que casi siempre la aseguraría en mayor medida sería la aplicación formalista de reglas.

Por eso me parece mucho más decisivo discutir el primero de aquellos dos pasos. Si el imperio de la ley es solo un ingrediente del ideal más complejo del Estado de Derecho $^{3}$, o solo uno de los componentes que integran la idea de justicia ${ }^{4}$, entonces otros aspectos del Estado de Derecho o de la justicia podrían requerir, para asegurar otros objetivos distintos de la predecibilidad pero igualmente valiosos y potencialmente en conflicto con ella, que no todas las normas jurídicas se formulen como reglas o que las formuladas como tales no siempre se apliquen de manera formalista ${ }^{5}$. En realidad la propia idea de autonomía requiere, sin duda, más cosas que la predecibilidad, de manera que si en algún sentido el valor de la autonomía personal exige la aplicación formalista de reglas, en otros puede exigir precisamente apartarse de ese modelo. Porque no hay autonomía si ciertas esferas de mi vida, ciertos intereses fundamentales para mí, no están protegidos frente a la acción o la inacción de terceros, esto es, sin el reconocimiento de derechos ${ }^{6}$, cuya efectividad requerirá la imposición sobre los de-

1 Este núcleo argumental, desarrollado minuciosamente en LAPORTA, 2007, aparece ya desde LAPORTA, 1994. Por supuesto, la predecibilidad requeriría además que esas normas fuesen relativamente estables, no retroactivas, etcétera.

2 Vid. Lifante, 2013. Partiendo de que la previsibilidad puede alcanzarse en distintos grados, no sería condición necesaria porque la forma en que se vayan a ponderar principios, a interpretar conceptos valorativos o a llevar a cabo interpretaciones amplificadoras o restrictivas de reglas en atención a las razones subyacentes, no tendría por qué ser — si existen convenciones asentadas al respecto- del todo imprevisible. Y tampoco sería condición suficiente, porque si la regulación de cierto ámbito de conducta toma la forma de un número muy elevado de reglas sumamente prolijas, podría suceder que lo que realmente permita predecir con mayor éxito el contenido de las decisiones de los órganos aplicadores sea el conocimiento de los principios subyacentes a esa esfera de regulación y una expectativa fundada de que en todo caso esa masa de reglas se interpretaría y aplicaría de manera congruente con aquellos.

3 LAPORTA, 1994: 138; 2002: 127; 2012: 88.

4 LAPORTA, 2002: 127; 2007: 83.

5 Tras la publicación de El imperio de la ley lo apuntaron enseguida FERREREs, 2009: 418-419; RODILLA, 2009: 41-43; o RUIZ MANERO, 2009: 109-116. Recientemente —-desarrollando ideas defendidas en trabajos precedentes- y en sentido similar, vid. MORESO, 2016. MORESO muestra persuasivamente que, al no poder considerar ningún legislador todas las combinaciones posibles de propiedades relevantes, la aplicación formalista de reglas entrañará en algunos casos el sacrificio de la justicia formal (tratar del mismo modo los casos iguales en los aspectos relevantes); y que también la justicia formal es una exigencia del ideal del Estado de Derecho y apartarse de ella una forma más de arbitrariedad.

6 Lo que, naturalmente, siempre han enfatizado LAPORTA (2007: 24 y ss.; 2012: 99) y HIERRO (2016a: 54). 
más de prohibiciones y obligaciones ${ }^{7}$. Pero no parece fácil concretar exhaustivamente en forma de reglas (con autonomía semántica y llamadas a ser aplicadas de manera formalista) el contenido, alcance y límites de esos derechos. E incluso formular y aplicar de esa manera aquellas prohibiciones y obligaciones que han de pesar sobre los demás puede, en ciertos casos, ser contraproducente, si lo que pretendemos es que sus conductas acaben ajustándose tanto como sea posible a lo que realmente exigiría el respeto de aquellas esferas protegidas ${ }^{8}$. Así que, si nos importa la autonomía, la predecibilidad que favorece la aplicación formalista de reglas tiene un valor, pero también unos costes. Y parece que un balance apropiado entre ellos ${ }^{9}$ requeriría más bien un derecho con reglas, pero no solo de reglas, y en el que no todas las reglas se apliquen necesariamente de manera formalista.

2. No creo, sin embargo, que la única alternativa a un juez formalista sea esa clase de «juez principialista» que LAPORTA no ha dudado en calificar de «peligro público» (LAPORTA, 2011: 178). En mi opinión, lo que requiere el imperio de la ley es un juez deferente. Pero para aclarar qué habría que entender por tal y en qué se diferenciaría ese tipo de juez del formalista y el principialista conviene reconsiderar qué valores subyacen al ideal poliédrico del imperio de la ley.

HIERRO (1996: 288-289) distinguió tempranamente dos interpretaciones del mismo: una débil —que atribuía a LAPORTA, 1994—, según la cual la sujeción a la ley de los órganos aplicadores sería importante, esencialmente, para que sus decisiones no resultaran impredecibles; y otra fuerte — que él suscribía — que, poniendo en primer plano las cuestiones de legitimidad y distribución del poder y mirando por tanto hacia el origen de la ley y no meramente hacia su forma, entendería que la sujeción a la ley de los órganos aplicadores es importante, esencialmente, para que el contenido de sus decisiones sea reconducible a la voluntad de quien ostenta la autoridad legítima. En el desarrollo ulterior de sus ideas la diferencia entre HIERRO y LAPORTA resulta ser solo de acento, puesto que ambos reconocen el valor inherente a cada una de esas interpretaciones del imperio de la ley ${ }^{10}$. Pero creo que de una y otra pueden derivarse visiones diferenciadas de la interpretación y aplicación del derecho o, si se quiere, distintos modelos de juez ${ }^{11}$.

Para un juez formalista, una regla soluciona normativamente todos los casos individuales subsumibles en su supuesto de hecho, sin resquicio para abrir el razonamiento aplicativo a la consideración de las razones subyacentes ni por tanto para resolver, ape-

\footnotetext{
${ }^{7} \mathrm{O}$, lo que viene a ser igual, la introducción de excepciones a lo que otras normas les permitirían hacer o dejar de hacer.

8 Vid. ENDICOTT, 2011: 27; WaLDRON, 2011: 75-81. Como los comportamientos que deberían evitarse no pueden ser concretados exhaustivamente en forma de reglas, formular de ese modo los correspondientes deberes - y aplicar esas reglas de manera formalista — permitiría a sus destinatarios explotar las lagunas de regulación en perjuicio de los derechos de los demás. En cambio, una regulación menos precisa (mediante cláusulas generales que recurran a conceptos valorativos), existiendo indicadores incompletos pero suficientes del sentido en que se aplicaría, alcanzaría el efecto inhibidor óptimo de los comportamientos indeseados precisamente por ser solo parcial la previsibilidad. Lo ilustra espléndidamente PAZ-ARES, 2015, con referencia al modo en que la Ley 31/2014 regula los deberes de lealtad de los administradores de las sociedades de capitales.

9 Vid. SHAPIRO, 2011: 258; ENDICOTT, 2011: 28 y 30.

10 Vid. HierRo, 1996: 289; 2016b: 190; y LAPORTA, 1999: 323, 328; 2002: 106-107; 2007: 144-147, 151.

${ }^{11}$ Me refiero siempre al juez ordinario.
} 
lando a dichas razones, ninguno de aquellos casos de manera diferente a lo dispuesto literalmente en su formulación. Las reglas son para el formalista generalizaciones estrictamente atrincheradas. Por el contrario, el juez principialista que nos dibujan HIERRO y LAPORTA — para denostarlo con razón — no solo no ve nunca en la ley una pauta llamada a controlar de manera concluyente sus decisiones, que para él han de resultar directamente de la ponderación —su ponderación — de las razones en juego, sino que además no toma en serio que dichas razones hayan de ser las del legislador, las que este haya tenido en cuenta y con el peso que haya pretendido darles. Más bien, alzando la mirada por encima de la ley, pretende recabarlas de su lectura de la constitución (indistinguible con frecuencia de su idea de justicia), con lo que en definitiva sustituye lo que el legislador ha decidido por lo que él estima que debería haber decidido. Que al hacerlo comprometa también la predecibilidad puede ser solo un modo más —y no el más grave- en el que ofende el ideal del imperio de la ley.

El juez deferente profesa la lealtad a la ley, pero no la entiende como el formalista. Si la aplicación estricta de las reglas lleva a resultados contrarios a sus razones subyacentes, entiende que el legislador no ha querido dichos resultados y que la auténtica fidelidad a la ley consiste en decidir atendiendo a esas razones. Pero si va a hacer tal cosa, a diferencia del principialista se toma muy en serio que le incumbe la carga de justificar en qué sentido podría afirmarse que esas son las razones del legislador y no meramente las suyas. Y acepta también que, sin serlo, tendrá que aplicar algunas reglas como lo haría un formalista: siempre que quepa sostener que entre las razones del legislador está precisamente la consideración de que para esa clase de casos la certeza de la decisión es más importante que su perfecto ajuste a las razones sustantivas subyacentes; o la de que, para esa clase de casos, a la larga las decisiones de los órganos aplicadores se aproximarán más a lo que realmente justificarían aquellas razones sustantivas si aplican mecánicamente la regla que si intentan determinar por sí mismos caso por caso qué sería acorde con dichas razones ${ }^{12}$.

Creo que el modelo del juez deferente es el que mejor se ajusta a las distintas dimensiones del ideal del imperio de la ley, tal y como nos las presentan los propios HIERRO y LAPORTA. Que a la luz de ellas reivindiquen en cambio el formalismo ${ }^{13}$ se debe fundamentalmente a que se pone en duda que pueda hablarse con sentido de la intención del legislador o que sea viable la identificación objetiva de las razones subyacentes a las reglas que ha dictado ${ }^{14}$. Y si no caben tales cosas, un juez que se dice deferente sería en realidad indistinguible de un juez principialista. Este es un punto crucial que aquí solo es posible abordar apresurada y superficialmente. Por supuesto una comprensión ingenua de la noción de «intención del legislador» (como el contenido de un estado mental de una suerte de mente colectiva) es descartable sin más al estar viciada por insuperables problemas tanto ontológicos como epistémicos. Cosa distinta es que no quepa reconstruirla conceptualmente de otro modo, a partir, por

12 Con terminología de SHAPIRO, lo que asumiría un juez deferente es en suma que debe actuar siendo fiel en cada caso a la «economía de la confianza» del sistema (SHAPIRO, 2011: 335-336, 355, 382, 398).

13 Expresa y pormenorizadamente en LAPORTA, 2007; más brevemente y tal vez con matices, HIERRO, 1996: 301-303, 306; 2016b: 198-204.

${ }_{14}$ Puede concederse sin dificultad que en ocasiones no será viable. Distinto es, naturalmente, sostener que nunca lo es. 
un lado, de una reconsideración del papel de las intenciones en la determinación del significado (entendiendo que al decir algo no es posible «querer decir» con ello sino lo que se entendería normalmente que se ha dicho dado el conjunto de convenciones de todo tipo presupuestas en ese contexto) y, por otro, de una elaboración aceptable de la idea de agencia colectiva (que no presuponga «entes colectivos», sino entramados complejos de reglas de imputación) ${ }^{15}$. Pero ni siquiera hace falta avanzar más por esa vía. LAPORTA afirma expresamente que aunque no quepa hablar de «intención del legislador», sí cabe hacerlo de «propósito de la ley», que no sería sino la justificación subyacente a toda regla; y que, aunque su identificación puede no ser sencilla y hay que cuidarse de no confundirla con una mera proyección del intérprete, existen criterios - básicamente de coherencia- que la hacen controlable ${ }^{16}$. No creo que la idea de juez deferente necesite más para abrirse paso, haciendo a un lado al juez formalista que estaba ocupando inmerecidamente el primer plano.

$$
* * *
$$

Más de una vez le he oído a Francisco LAPORTA recordar el viejo dicho de GINER: «Lo que sabemos, lo sabemos entre todos». Siempre me gustó esa frase. Pero que de nadie provenga en exclusiva todo lo que entre todos sabemos no quiere decir, naturalmente, que todos hayamos podido contribuir a ese fondo común en igual medida. Quienes hemos tenido la suerte de convivir y trabajar con Curro y Paco, de aprender de ellos, somos muy conscientes de que gran parte de las ideas que hoy son nuestras fueron antes suyas. Y sabemos también que siempre quisieron a su lado compañeros críticos y nunca han dejado de estar dispuestos a hacer suya una idea nuestra si es que llegaba a parecerles convincente. Que siga siendo así por muchos años.

\section{BIBLIOGRAFÍA}

EKINS, R., 2012: The Nature of Legislative Intent, Oxford: Oxford University Press.

ENDicotT, T., 2011: «The Value of Vagueness», en MARMor y SOAmEs (eds.), 14-30.

FerReres, V., 2009: «El imperio de la ley y sus enemigos», Revista Española de Derecho Constitucional, 87: 413-426.

HIERRO, L., 1996: «El imperio de la ley y la crisis de la ley», Doxa, 19: 287-308.

- 2016a: Los derechos humanos. Una concepción de la justicia, Madrid: Marcial Pons.

- 2016b: «Seguridad jurídica, crisis de la ley y crisis de la aplicación de la ley», en L. PEÑa y T. Ausín (coords.), Conceptos y valores constitucionales, Madrid: Plaza y Valdés, 187-208.

LAPORTA, F. J., 1994: «Imperio de la ley. Reflexiones sobre un punto de partida de Elías Díaz», Doxa, 15-16/I: 133-145.

- 1999: «Materiales para una reflexión sobre la racionalidad y la crisis de la ley», Doxa, 22: $321-330$.

— 2002: «Imperio de la ley y seguridad jurídica», en E. DíAz y J. L. Colomer (eds.), Estado, justicia, derechos, Madrid: Alianza, 105-132.

15 En esa línea, vid. Ekins, 2012.

16 LAPORTA, 2007: 2016; 2012: 118. En realidad LAPORTA limita la apelación al propósito de la ley a los supuestos de indeterminación del derecho. Pero si cabe su identificación objetiva, no veo por qué habría de ser así. 
- 2007: El imperio de la ley. Una visión actual, Madrid: Trotta.

— 2011: «Sobre Luigi Ferrajoli y el constitucionalismo», Doxa, 34: 167-181.

— 2012: «El imperio de la ley: un diálogo en Génova», Analisi e Diritto, 87-121.

Laporta, F. J.; Ruiz MAnero, J., y Rodilla, M. Á.: Certeza y predecibilidad de las relaciones jurídicas, Madrid: Fundación Coloquio Jurídico Europeo.

LIFANTE, I., 2013: «Seguridad jurídica y previsibilidad», Doxa, 36: 85-105.

Marmor, A., y SoAmes, S. (eds.), 2011: Philosophical Foundations of Language in the Law, Oxford: Oxford University Press.

Moreso, J. J., 2016: «Marry Me A Little. How Much Precision Is Enough In Law?», manuscrito, en Academia.edu.

PAZ-Ares, C., 2015: «Anatomía del deber de lealtad», Actualidad Jurídica Uría-Menéndez, 39 : 43-65.

Rodilla, M. Á., 2009: «Imperio de la ley y principios. Presentación de un debate», en LAPORTA, RuIZ MANERO y RODILLA, 2009: 9-53.

Ruiz MANero, J., 2009: «Las virtudes de las reglas y la necesidad de los principios. Algunas acotaciones a Francisco Laporta», en LAPORTA, RuIZ MANERO y RODILLA, 2009: 95-122.

SHAPIRO, S., 2011: Legality, Cambridge, Mass.: Harvard University Press.

Waldron, J., 2011: «Vagueness and the Guidance of Action», en MARMOR y SOAmES (eds.), 2011: 58-82. 